\title{
Longitudinal vitamin and homocysteine levels in normal pregnancy
}

\author{
Rolf J. L. M. Cikot ${ }^{1}$, Régine P. M. Steegers-Theunissen ${ }^{1,2}$, Chris M. G. Thomas ${ }^{1,3}$, Theo M. de Boo ${ }^{4}$, \\ Hans M. W. M. Merkus ${ }^{1}$ and Eric A. P. Steegers ${ }^{1 *}$ \\ Department of ${ }^{1}$ Obstetrics and Gynaecology, ${ }^{2}$ Epidemiology, ${ }^{3}$ Chemical Endocrinology and ${ }^{4}$ Medical Statistics, University \\ Medical Centre St Radboud, 6500 HB Nijmegen, The Netherlands
}

(Received 24 May 1999 - Revised 20 June 2000 - Accepted 17 July 2000)

\begin{abstract}
Evidence of the impact of maternal nutritional status on pregnancy outcome is increasing. However, reference values for vitamin and homocysteine concentrations in maternal blood during normal pregnancy are scarce, and are lacking for the preconceptional period and early pregnancy. Thus, in a longitudinal study we evaluated vitamin and homocysteine concentrations in 102 nulliparous women with an uneventful singleton pregnancy and normal outcome not using supplements. The physiological changes in vitamin and homocysteine concentrations in blood were determined from the preconceptional period throughout pregnancy until 6 weeks post-partum. The vitamins evaluated comprised retinol, thiamin, riboflavin, pyridoxal $5^{\prime}-$ phosphate, folate in serum and erythrocytes, vitamin $\mathrm{B}_{12}$ and $\alpha$-tocopherol. The plasma homocysteine concentration was also measured, considering the essential roles of folate, vitamin $\mathrm{B}_{6}$ and vitamin $\mathrm{B}_{12}$ in homocysteine metabolism. The concentrations of retinol, thiamin, pyridoxal $5^{\prime}$-phosphate serum folate and vitamin $\mathrm{B}_{12}$ decreased during pregnancy. In contrast, the concentrations of riboflavin, $\alpha$-tocopherol, and folate in erythrocytes increased or showed only minor changes. Homocysteine concentrations also remained approximately constant during pregnancy. These observations emphasize the importance of preconceptional and post-partum concentrations of vitamins in the evaluation of pregnancy-induced changes. These data have provided valuable reference values for vitamins and homocysteine before, during and after pregnancy in order to contribute to better diagnosis of maternal deficiencies and to study further the relationship between maternal vitamin status and adverse course and outcome of pregnancy.
\end{abstract}

Vitamins: Homocysteine: Pregnancy: Nutritional status

Maternal nutritional status has been shown to contribute significantly to pregnancy outcome (Brown, 1993). Food intake, processing, absorption, metabolism and clearance determine the individual vitamin status. The embryo and fetus are totally dependent on the maternal-fetal transfer of nutrients and the maternal nutrient status. Thus, it can be expected that maternal nutritional deficiencies, especially periconceptionally, may influence the course and outcome of pregnancy. In addition, marginally deficient values or elevated concentrations still within the normal nonpregnant range might have a significant impact on early embryonic development as well as long-term health outcome. With respect to long-term health outcome, a relationship between low birth weight and insulin resistance and cardiovascular disease in adult life has been reported (Barker et al. 1993; Phillips et al. 1994).

The harmful effects of hypervitaminosis $\mathrm{A}$ and the beneficial effects of folate supplementation have already received much attention, which further emphasizes the need for reference values for these vitamins before and during normal pregnancy. With this information, diagnosis of maternal vitamin deficiencies and the evaluation of medical treatment can be improved. In addition, future studies on the relationship between maternal vitamin status and the occurrence of an abnormal course and outcome of pregnancy will become feasible.

Data on longitudinally-determined vitamin concentrations in normal pregnancy are scarce, and there is a lack of preconceptional and first trimester values (Bruinse \& van den Berg, 1995). Most available data are derived from cross-sectional studies in which vitamin concentrations are determined at various gestational ages (Baker et al. 1975). Moreover, the study populations concerned are mostly heterogeneous with regard to parity and ethnic descent.

The aim of the present study, therefore, was to determine in healthy women who were not using any vitamin or food supplements and had a normal pregnancy outcome, the blood concentrations of retinol, thiamin, riboflavin, pyridoxal $5^{\prime}$-phosphate, folate, vitamin $\mathrm{B}_{12}, \alpha$-tocopherol and the vitamin-dependent homocysteine concentration from

\footnotetext{
* Corresponding author: Dr Eric Steegers, fax + 3124354 1194, email e.steegers@obgyn.azn.nl
} 
the preconceptional period throughout pregnancy until 6 weeks after delivery.

\section{Experimental methods}

\section{Protocol}

As part of an extensive study on risk factors for adverse pregnancy outcome in epileptic women, a cohort of 225 women including healthy controls was formed in the period 1987-90. The design of this observational study has previously been described in detail (Steegers-Theunissen et al. 1994). The Medical Ethical Committee of the University Medical Centre St Radboud in Nijmegen, The Netherlands, approved the protocol. Women were recruited preconceptionally and were included after informed consent was obtained. Only nulliparous and singleton pregnancies were included in the present study. Before conception a research nurse visited the women at home every 6 months for history taking and blood sampling. Pregnancy was confirmed by a monoclonal antibody-based pregnancy test. Menstrual history, basal body temperature charting and one or two transvaginal ultrasonographic examinations before week 8 of gestation confirmed the duration of the pregnancy. Non-fasting random blood samples were taken within 3 months prior to conception and subsequently at $6,10,20$ and 32 weeks of gestation and 6 weeks post-partum.

Only women with an uneventful pregnancy course and normal outcome were included in the present study. Inclusion criteria for normal pregnancy were: no epilepsy; no pregnancy-induced hypertension or pre-eclampsia; no diabetes gravidarum; no hospital admission other than for labour; spontaneous labour between 37 and 42 weeks of gestation; birth weight appropriate for gestational age according to the Dutch birth weight curve (birth weight between the percentiles 2.3 and 97.7) (Kloosterman, 1970). The study population fulfilling the described criteria comprised 102 women.

The following vitamins were evaluated: retinol, thiamin, riboflavin, pyridoxal 5'-phosphate, folate, vitamin $\mathrm{B}_{12} ; \alpha$ tocopherol. Homocysteine was also measured, considering the essential role of vitamin $\mathrm{B}_{6}$, vitamin $\mathrm{B}_{12}$ and folate in homocysteine metabolism.

\section{Analytical procedures}

We have participated in external quality assurance programmes of The Netherlands Foundation for Quality Assurance in Clinical Laboratories (thiamin, vitamin $\mathrm{B}_{6}$, vitamin $\mathrm{A}$ and vitamin $\mathrm{E}$ ), the Landelijke workgroup Bindingsanalyse (vitamin $\mathrm{B}_{12}$ and folate) and the German programme. Deutsche Ringversuch of the German Society for Clinical Chemistry.

Serum retinol concentration was determined using HPLC with fluorescence detection, and retinal acetate as the internal standard. Before HPLC, the serum sample was denatured with methanol, extracted with $n$-hexane and the dried residue was taken up in ethanol. Fluorescence excitation and emission wavelengths were 348 and $470 \mathrm{~nm}$ respectively. The minimum detectable concentra- tion was $0.06 \mu \mathrm{mol}$ retinol/l and the precision in terms of intra- and inter-assay CV for means $(0.96 \mu \mathrm{mol} / \mathrm{l})$ of duplicate determinations were 1.1 and $5.3 \%$ respectively.

Plasma thiamin concentration was determined using reverse-phase ion-pair HPLC. Whole blood was haemolysed by freezing and serum was deproteinized using perchloric acid. Thiamine pyrophosphate and thiamine monophosphate were hydrolysed to thiamine by acid phosphatase. HPLC was performed using $\mathrm{C}_{18}$-coated silica with an $n$-octanesulfonate-containing mobile phase methanol-aqueous citrate buffer, $\mathrm{pH} 4 \cdot 0(45: 55, \mathrm{v} / \mathrm{v})$. In a postcolumn reaction $\mathrm{K}_{3} \mathrm{Fe}(\mathrm{CN})_{6}$ was used to oxidise thiamine to thiochrome, which was detected by fluorometry with excitation and emission wavelengths set at 364 and $462 \mathrm{~nm}$ respectively. The minimum detectable amount was $2.3 \mathrm{nmol}$ thiamine/l. At mean concentrations of $123 \mathrm{nmol} / \mathrm{l}$ the intra-assay $\mathrm{CV}$ was $4.8 \%$ and the interassay $\mathrm{CV}$ was $6.8 \%$.

Riboflavin was determined in heparinized plasma using HPLC and fluorescence detection. Before HPLC, heparinized whole-blood samples were denatured with trichloroacetic acid, centrifuged and the supernatant fraction injected onto the HPLC. Fluorescence excitation and emission wavelengths were 470 and $520 \mathrm{~nm}$ respectively. The minimum detectable concentration was $10 \mathrm{nmol}$ riboflavin/l, and the precision in terms of intra- and interassay $\mathrm{CV}$ for means $(90 \mathrm{nmol} / \mathrm{l})$ of duplicate determinations were 3.9 and $8.2 \%$ respectively.

The concentration of pyridoxal $5^{\prime}$-phosphate in whole blood was measured using HPLC. Before HPLC $1 \mathrm{ml}$ heparinized whole blood was denatured with perchloric acid, centrifuged and the supernatant fraction was neutralized with $\mathrm{KOH}$ and injected onto the HPLC column. Post-column derivatization was performed with semicarbizide reagent to obtain the pyridoxal $5^{\prime}$-phosphate semicarbazone. The fluorescence was determined using wavelengths for excitation and emission of 367 and $478 \mathrm{~nm}$ respectively. The minimum detectable concentration was 2 nmol pyridoxal $5^{\prime}$-phosphate/l and the precision in terms of intra- and inter-assay CV was $1.6 \%$ (twenty replicate determinations; mean concentration $52 \mathrm{nmol} / \mathrm{l}$ ) and $5.5 \%$ (fifteen determinations on several days; mean concentration $53 \mathrm{nmol} / \mathrm{l}$ ) respectively.

Vitamin $B_{12}$ and folate concentrations in serum were determined simultaneously with the dualcount solid phase boil radioassay (Diagnostic Products Corporation, Los Angeles, CA, USA). Folate was also determined in erythrocytes (Dunn \& Foster, 1973) and $24 \mathrm{~h}$ urine samples. The radioassay makes use of a dual-label tracer solution ( $\left[{ }^{57} \mathrm{Co}\right]$ cyanocobalamin and ${ }^{125}$ I-labelled folic acid) and a suspension of the solid-phase binders for vitamin $B_{12}$ and folate. After incubation and bound-free separation by centrifugation, the radioactivity of the bound fraction was counted and the concentrations of vitamin $\mathrm{B}_{12}$ and folate were calculated from logit-log transformation of the data to obtain linear standard dose-response curves (with the logit being the natural logarithm of binding responses). Assay precision in terms of intra- and interassay CV for means of duplicate determinations were $<5$ and $10 \%$ respectively for both vitamins.

$\alpha$-Tocopherol concentrations were determined in serum 
by HPLC with fluorescence detection. The serum samples were denatured with ethanol, diluted with twice-distilled water and centrifuged after addition of the mobile phase ( $n$ hexane-ethanol 99.5:0.5, v/v). Using an automated sampler, $20 \mu \mathrm{l}$ supernatant fraction was injected onto a normal phase guard column which was in line with a silica column and equipped with an automatic HPLC liquid solvent pump. The flow rate was $2.0 \mathrm{ml} / \mathrm{min}$ and the fluorescence excitation and emission wavelengths for $\alpha$-tocopherol were 295 and $390 \mathrm{~nm}$ respectively. The minimum detectable concentration was $0.40 \mu \mathrm{mol} \alpha$-tocopherol/l. The intraand inter-assay $\mathrm{CV}$ were $2.8 \%$ (ten replicate determinations; mean concentration $22.2 \mu \mathrm{mol} / \mathrm{l}$ ) and $6.6 \%$ (thirteen simultaneous assay runs; mean concentration $23.7 \mu \mathrm{mol} / \mathrm{l}$ ), respectively.

Homocysteine concentrations in plasma were determined using HPLC with reverse-phase separation and fluorescence detection. Total homocysteine determination by this method is based on complete reduction of all homocystine disulfide bonds in plasma by $\mathrm{NaBH}_{4}$ and dithioerythritol. After derivatization by monobromobimane the resulting homocysteine-monobromobimane complex is separated from interfering substances by reverse-phase HPLC. The intra- and inter-assay CV were $2 \cdot 1$ and $5.2 \%$ respectively.

\section{Statistical analysis}

To estimate the longitudinal values of percentiles 5, 50 and 95 from the preconceptional period until 10 weeks postpartum a linear mixed model for repeated measurements was used. Three fixed parameters were used in this model: (1) the overall intercept; (2) the duration of gestation at a given time of measurement; (3) the duration squared. To allow for individual variation, three random parameters were added to the model: (1) the individual intercept; (2) the individual coefficient of duration; (3) the individual coefficient of duration squared. The covariance matrix was left completely unspecified. Except for post-partum values, all measurements were used for estimations within these models. For computational purposes, preconceptional values were used with duration set equal to zero.

The 50th percentile was estimated using the fixed coefficients calculated from the model. Variance estimators for the 50th percentile for individual variation and residual variance were added. The resulting standard deviation was used to compute the 5th and 95th percentiles. If on inspection residuals from the model appeared to be nonnormally distributed, a log- or square-root transformation was used on the original variables, the centiles were computed and then transformed back. The only exception to this procedure was for homocysteine, which was measured in a small subgroup of sixteen women. After $\log$ transformation, the 50th percentile was computed as the mean, the (total) standard deviation was multiplied by $1+$
$(1 / n)$, where $n$ is the number of measurements available at the given time, and this value was then used to compute the 5th and 95th percentiles; these values were then transformed back.

The analysis used the SAS procedure MIXED (Statistical Analysis Systems Institute Inc., Cary, NC, USA).

\section{Results}

Figs. 1-3 show the median values and the upper $\left(95^{\text {th }}\right)$ and lower $\left(5^{\text {th }}\right)$ percentiles of the concentrations of vitamins and homocysteine determined from preconception, throughout pregnancy, to 6 weeks post-partum.

Retinol (Fig. 1(a)) concentrations during pregnancy showed only a minor decrease. Post-partum concentrations were markedly elevated compared with preconceptional values.

Thiamin (Fig. 1(b)) concentrations showed a gradual decline. At 6 weeks post-partum preconceptional concentrations were reached.

Riboflavin (Fig. 1(c)) concentrations showed only minor changes during pregnancy, with a slight increase in the third trimester.

Pyridoxal 5'-phosphate (Fig. 1(d)) concentrations gradually declined during pregnancy. Post-partum values were similar to those found preconceptionally.

Serum folate (Fig. 2(a)) concentrations showed a slight decrease during pregnancy and remain decreased up to 6 weeks after delivery. Folate concentrations in erythrocytes (Fig. 2(b)) increased slightly during pregnancy. In the postpartum period erythrocyte folate concentrations were lower than those in the third trimester, but similar to preconceptional concentrations. Fig. 2(c) shows the $24 \mathrm{~h}$ secretion of folate in urine which does not seem to change throughout pregnancy. Also no differences were observed regarding folate excretions in the puerperium.

Vitamin $\mathrm{B}_{12}$ (Fig. 1(e)) concentrations showed a progressive decline during pregnancy reaching marginal or even deficient levels. At 6 weeks after delivery vitamin $\mathrm{B}_{12}$ reached preconceptional values.

$\alpha$-Tocopherol (Fig. 1(f)) concentrations during pregnancy showed a progressive increase when compared with preconceptional values. After delivery $\alpha$-tocopherol concentrations returned to the preconceptional values.

Homocysteine (Fig. 3) concentrations declined slightly in the first trimester and remained approximately constant during pregnancy. Post-partum concentrations were slightly elevated compared with preconception values.

\section{Discussion}

The present study is the first longitudinal study in which vitamin and homocysteine concentrations in blood have been determined from the preconceptional period through

Fig. 1. Serum concentrations of (a) retinol, (b) thiamin, (c) riboflavin, (d) pyridoxal $5^{\prime}$-phosphate, (e) vitamin $B_{12}$ and (f) $\alpha$-tocopherol in 102 nulliparous Dutch women from the preconceptional period, throughout a normal healthy pregnancy, to 6 weeks post-partum. Samples were taken within the 3 months before conception and subsequently at weeks $6,10,20$ and 32 of gestation and at 6 weeks post-partum, with gestation shown as the duration weeks of amenorrhoea (weeks). Individual values $(\bigcirc)$ and the 5th (. - . -), 50th (-) and 95th (- - - ) percentiles are shown. For details of subjects and procedures, see p. 50 . 

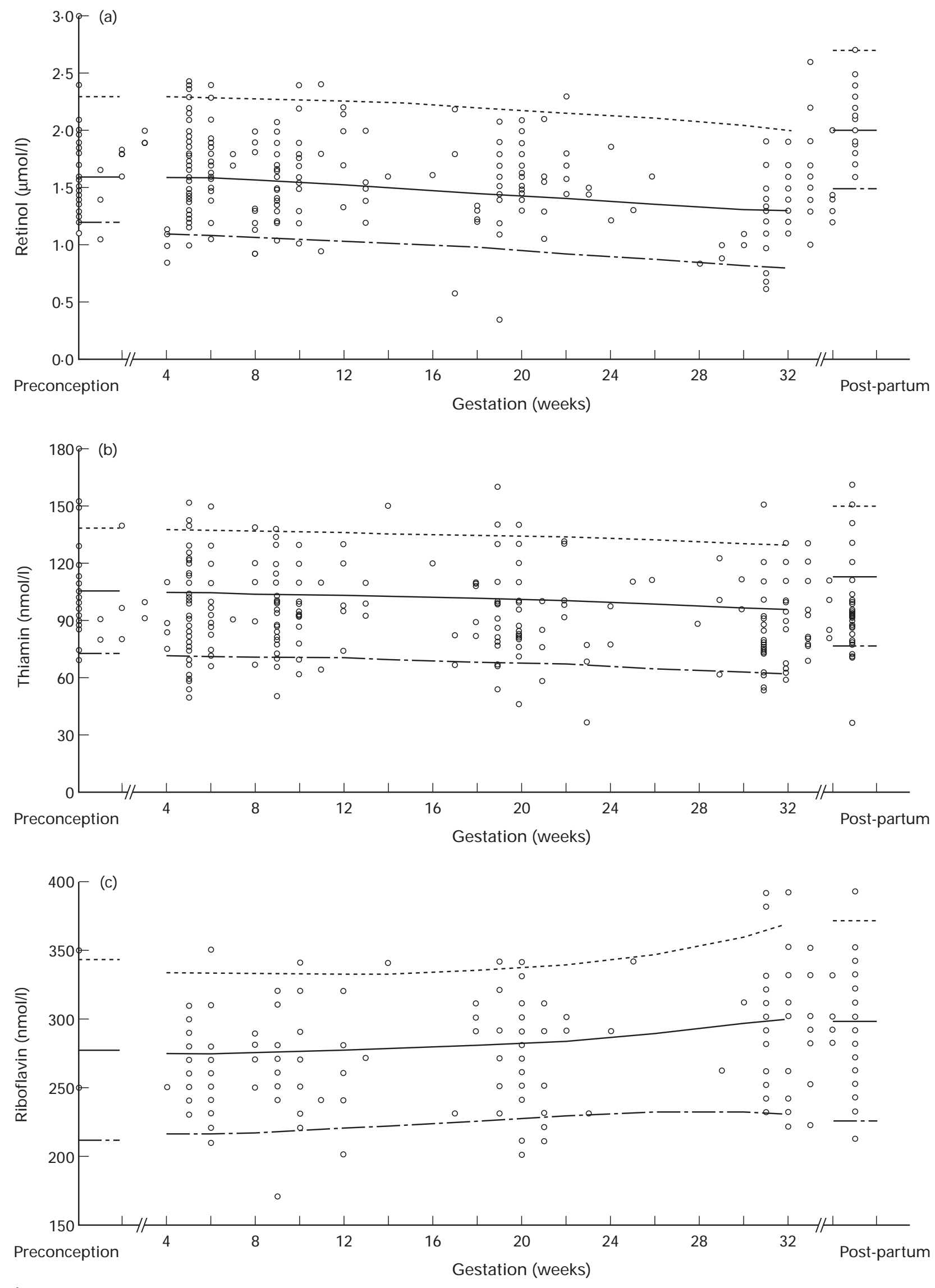

Fig. 1. 

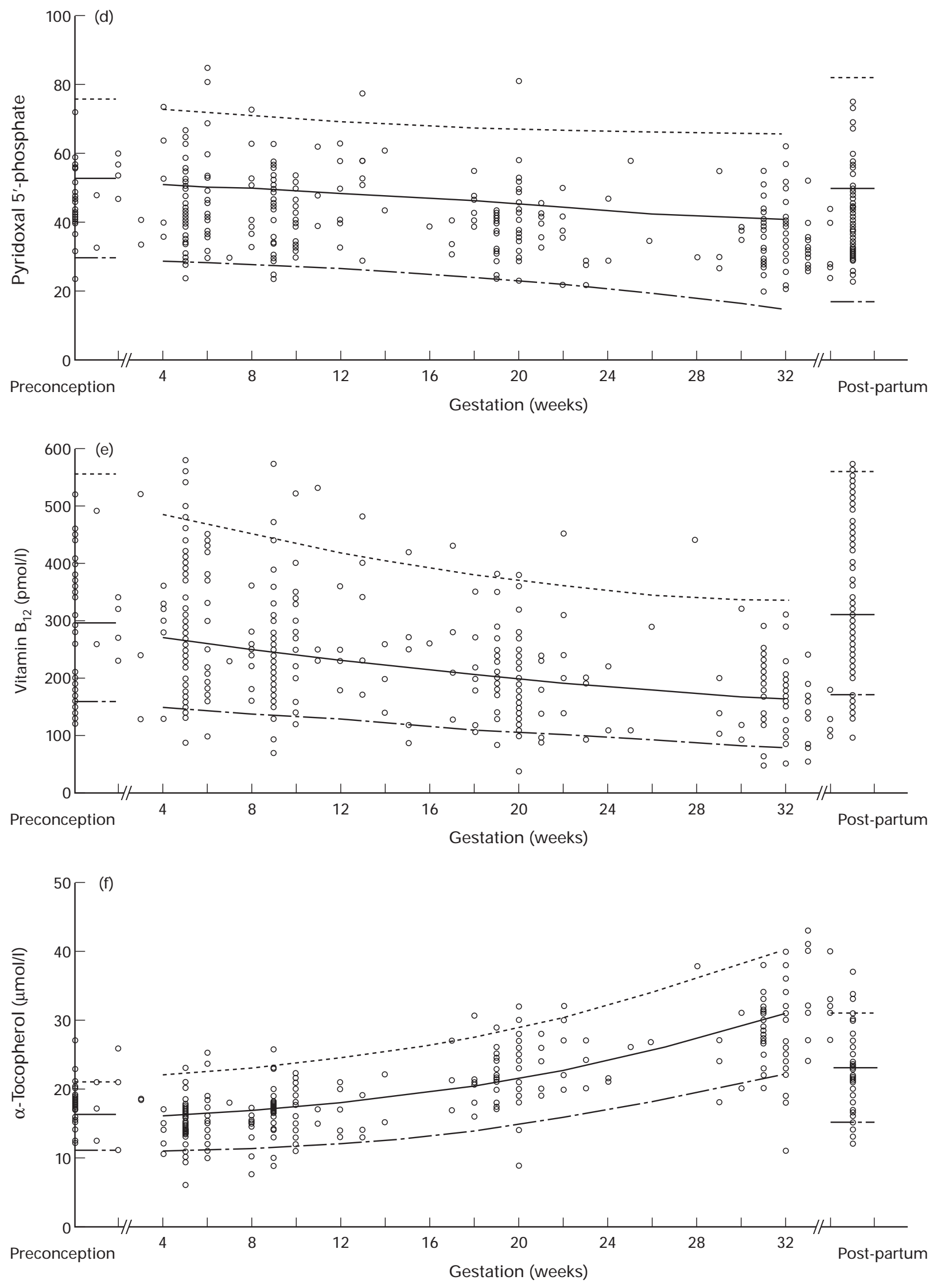

Fig. 1. Continued 

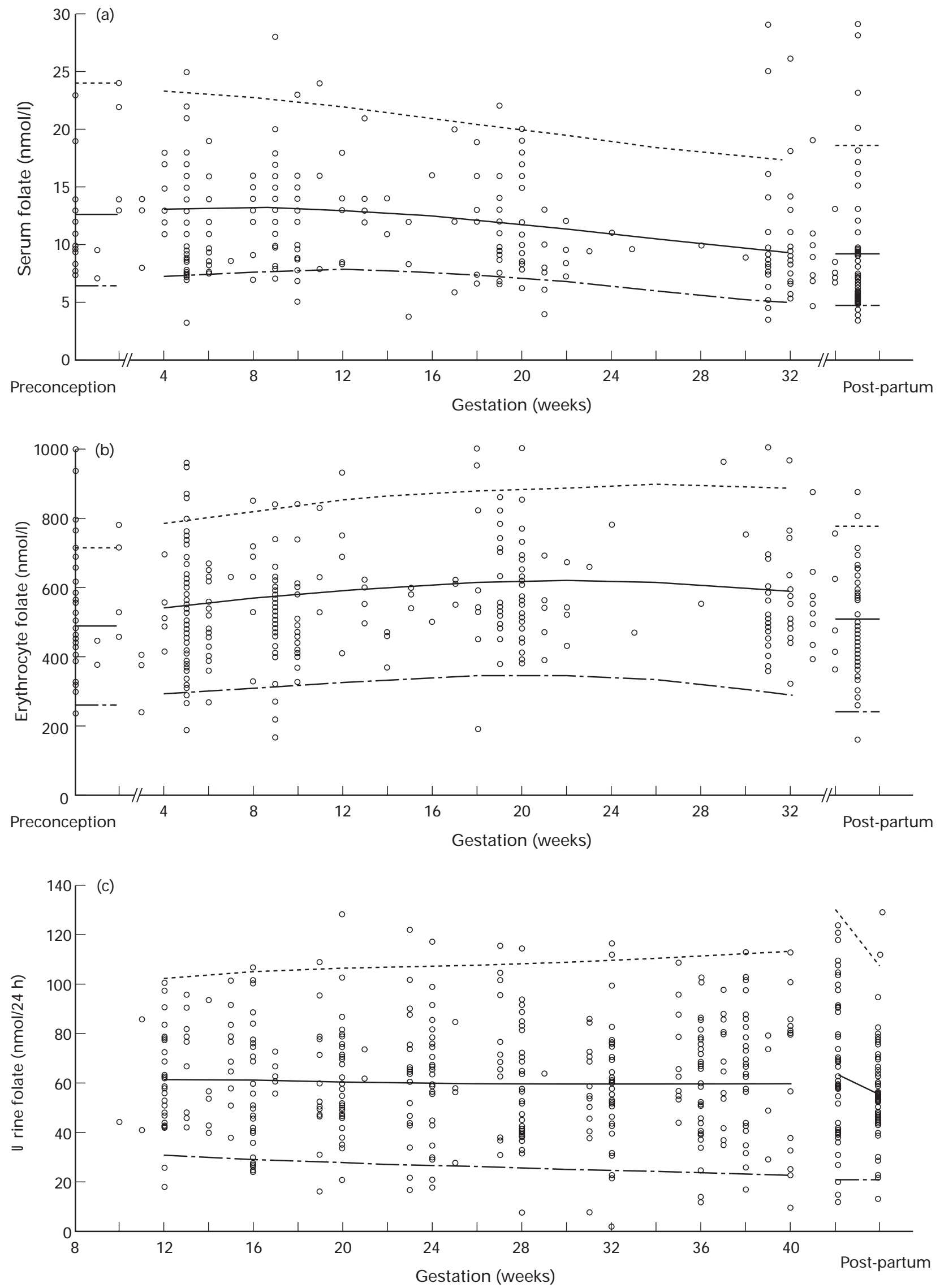

Fig. 2. 


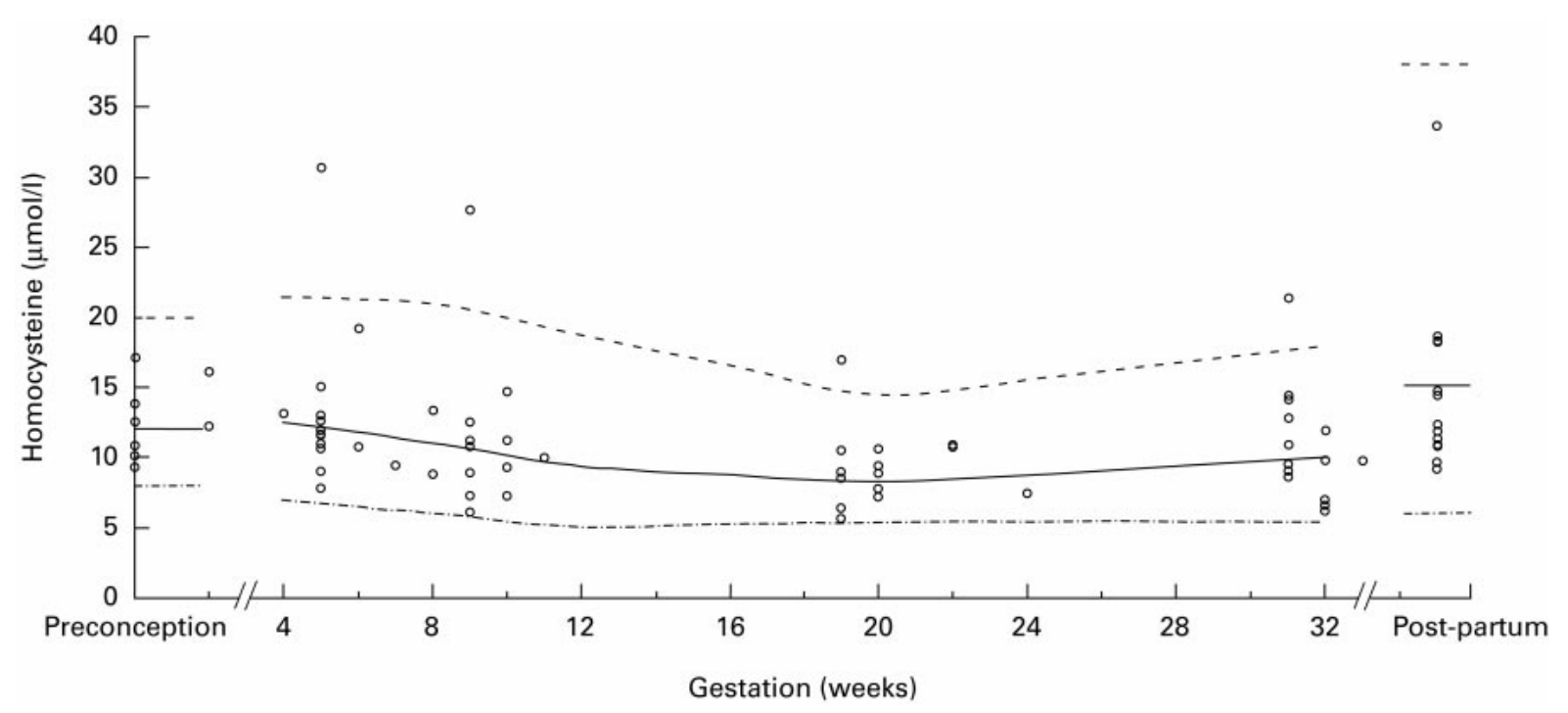

Fig. 3. Plasma homocysteine concentrations in sixteen nulliparous Dutch women from the preconceptional period, throughout a normal healthy pregnancy, to 6 weeks post-partum. Samples were taken within the 3 months before conception and subsequently at weeks $6,10,20$ and 32 of gestation and at 6 weeks post-partum, with gestation shown as the duration of amenorrhoea (weeks). Individual values $(O)$ and the 5 th $(\cdot-\cdot-)$, 50th (-) and 95th (- - -) percentiles are shown. For details of subjects and procedures, see p. 50.

pregnancy, to the post-partum period, in a homogeneous population of nulliparous Dutch women who did not use vitamin supplements or supplemented diets. The present study has provided valuable reference values for these micronutrients, which are representative for healthy women aged 20-40 years with an uncomplicated singleton pregnancy and normal pregnancy outcome.

The minor decrease in vitamin A during pregnancy and the elevated concentrations after delivery are in accordance with previous studies (Baker et al. 1975; Bruinse \& van den Berg, 1995). However, other researchers have reported an increase during pregnancy (Gal \& Parkinson, 1974). Tissue retention of the fat-soluble vitamin A during pregnancy and weight loss after delivery may account for the observed changes. Vitamin A and its precursors can readily move across the placenta. Hypervitaminosis A is teratogenic (Lammer et al. 1985; Rothman et al. 1995) resulting in congenital abnormalities and a high incidence of spontaneous abortions. Vitamin A is an important antioxidant and significantly lower concentrations have been reported in pregnancies complicated by pre-eclampsia (Ziari et al. 1996).

The thiamin status did not seem to be affected by pregnancy, showing only a gradual decline. Previous studies on transketolase activity in erythrocytes in well-nourished European women showed an insufficient thiamin reserve in one-third of the investigated population (Heller et al. 1974b; Nicols \& Nicols, 1983). Insufficient thiamin intake during pregnancy and lactation as well as hyperemesis gravidarum can lead to symptoms of deficiency, eventually leading to beri beri (Dyckner et al. 1985).
Riboflavin concentrations were also relatively unaffected by pregnancy, showing $<5 \%$ increase in the third trimester. Other researchers have reported similar results (Decker et al. 1977; Bruinse \& van den Berg, 1995). Riboflavin deficiency is associated with elevated glutathionine reductase activity in erythrocytes after in vitro incubation with riboflavin (Nicols \& Nicols, 1983). In a normal pregnancy the activity of this enzyme shows a $20 \%$ increase in the presence of riboflavin. On this basis, $20-40 \%$ of the pregnant women investigated by Heller et al. (1974a) were reported to be riboflavin deficient.

Several studies have reported a similar gradual decline in vitamin $\mathrm{B}_{6}$ concentrations (Lumeng et al. 1976). The decrease is suggested to occur mainly before the 16th week of gestation (Bruinse \& van den Berg, 1995). In the present study post-partum concentrations were similar to preconceptional values. At 6 months after delivery $25 \%$ of the serum vitamin $B_{6}$ concentrations were reported to be below the reference values for non-pregnant women (Bruinse \& van den Berg, 1995). This finding indicates that there is maternal vitamin $\mathrm{B}_{6}$ depletion during pregnancy, with preferential tissue store depletion. Vitamin $\mathrm{B}_{6}$ deficiency may lead to hyperhomocysteinaemia, which is an established risk factor for early-onset atherosclerosis and ischaemic vascular pathology (Boers et al. 1985) and adverse pregnancy outcome (see p. 56).

Folate is stored during erythropoiesis in erythrocytes. The folate concentration in erythrocytes is less dependent on dietary fluctuations than serum concentrations and is therefore a better indicator of folate status. Most studies indicate only minor changes or a slight decrease in the

Fig. 2. Concentrations of (a) serum folate, (b) erythrocyte folate and (c) urine folate in 102 nulliparous Dutch women from the preconceptional period, throughout a normal healthy pregnancy, to 6 weeks post-partum. Samples were taken within the 3 months before conception and subsequently at weeks $6,10,20$ and 32 of gestation and at 6 weeks post-partum, with gestation shown as the duration of amenorrhoea (weeks). Individual values $(O)$ and the 5th $(\cdot-\cdot-)$, 50th $(-)$ and 95th $\left(--_{-}\right)$percentiles are shown. For details of subjects and procedures, see p. 50. 
concentrations of folate in erythrocytes (Rolschau et al. 1979; Bruinse \& van den Berg, 1995). In the present study the concentrations of folate in erythrocytes initially increased slightly during pregnancy, but showed a minor decrease in the third trimester. At 6 weeks post-partum the erythrocyte folate concentrations returned to the preconceptional values. Serum folate concentrations showed a slight decrease during pregnancy, with recovery after delivery. Similar findings have been reported in almost all studies (Rolschau et al. 1979; Ek \& Magnus, 1981; Bruinse \& van den Berg, 1995). Our data confirm that serum folate concentrations remain decreased within 6 weeks after delivery. The results of a previous study indicated a marginal or even deficient state with respect to serum folate concentrations up to 6 months after delivery (Bruinse \& van den Berg, 1995). Possible explanations are the physiological haemodilution during pregnancy, lactation, hormonal influences and contraception or elevated urinary excretion (McPartlin et al. 1993). Folate deficiency has long been reported in association with adverse pregnancy outcome (Hibbard, 1964) and congenital abnormalities, especially neuraltube defects (Kirke et al. 1993). More recent data have confirmed the beneficial effect of periconceptional folate supplementation in the prevention of the occurrence and recurrence of neural-tube defects (Steegers-Theunissen et al. 1993). It has been suggested that either decreased folate intake or genetic disturbances in folate metabolism contribute to these conditions (Steegers-Theunissen et al. 1991; van der Put et al. 1995). The crucial role of folate, as a substrate, in homocysteine metabolism has been established.

The progressive decline of vitamin $B_{12}$ concentrations by about $100 \mathrm{pmol} / \mathrm{l}$ during pregnancy has been reported previously (Bruinse \& van den Berg, 1995). This decline leads to marginal or even deficient concentrations, which recover after pregnancy, and return to preconceptional values at 6 weeks post-partum. Compared with folate, possible relationships between vitamin $\mathrm{B}_{12}$ deficiency and congenital abnormalities are less clear. However, low or deficient serum and amniotic fluid concentrations of vitamin $\mathrm{B}_{12}$ have been associated with neural-tube defects (Steen et al. 1998). Additional studies are needed to elucidate the role of vitamin $\mathrm{B}_{12}$ in the prevention of adverse pregnancy outcome. So far, there is increasing evidence of a synergistic role of folate and vitamin $B_{12}$ in homocysteine metabolism (Bronstrup et al. 1998). Maternal megaloblastic anaemia, the result of vitamin $\mathrm{B}_{12}$ or folate deficiency, does not seem to affect the fetus. High cord blood concentrations suggest active transfer of vitamin $\mathrm{B}_{12}$ and folate across the placenta.

Vitamin E concentrations during pregnancy progressively increased compared with preconceptional values. This finding is in accordance with previous reports (Mooij et al. 1993). The increase in vitamin $\mathrm{E}$ might have a compensatory effect on the increase in oxygen radical formation as gestation progresses (Wang et al. 1991). The elevation of vitamin $\mathrm{E}$ seems to correspond with the increase in products of membrane damage with advancing duration of gestation. Fetal growth retardation and preeclampsia have been correlated with relatively low maternal concentrations of vitamin E (Von Mandach et al. 1994; Ziari et al. 1996).

A previous study revealed a significantly lower homocysteine concentration at the end of the first trimester compared with subsequent constant concentrations until delivery (Andersson et al. 1992). Our data confirm the slight decline in homocysteine concentrations in the first trimester. The homocysteine level remained approximately constant during the second and third trimester and was slightly lower than that of non-pregnant women. Post-partum values were slightly elevated compared with preconceptional concentrations. The changes in homocysteine metabolism during pregnancy may be explained by hormonal influences, haemodilution and the increased fetal and maternal needs for methionine and homocysteine (Steegers-Theunissen et al. 1997). Deficiencies of folate, vitamin $\mathrm{B}_{12}$ and vitamin $\mathrm{B}_{6}$ or genetic defects in their metabolism, may result in mildly elevated homocysteine concentrations in blood and urine. The fact that the homocysteine level fell as pregnancy progressed while folate, vitamin $\mathrm{B}_{12}$ and pyridoxal $5^{\prime}$-phosphate levels were also falling, might be explained by the strong effect of haemodilution on all these determinants. The decreased vitamin levels would otherwise be accompanied by a rise in the homocysteine concentration. During pregnancy an increased homocysteine level is a risk factor for recurrent spontaneous abortion, intra-uterine death, abruptio placentae and neural-tube defects (SteegersTheunissen et al. 1991, 1992; Kirke et al. 1993). It is not clear whether hyperhomocysteinaemia is a primary cause of these diseases and abnormal outcomes or is just an epiphenomenon.

Concentrations of the vitamins evaluated were measured in maternal serum or plasma. It is uncertain whether these concentrations represent vitamin functions at a cellular level, especially during pregnancy when an early shift from serum to tissue is proposed (Bruinse \& van den Berg, 1995). Early preconceptional data were, however, lacking in Bruinse \& van den Berg's report. Our data do not substantiate such a serum to tissue shift early in pregnancy. Most observed pregnancy-induced vitamin changes occur progressively during pregnancy. The mechanisms for the changes of vitamin concentrations during pregnancy have not yet been resolved. Increased renal excretion based on elevated glomerular filtration rate has been suggested (Bruinse \& van den Berg, 1995). Hormonal influences and haemodilution may contribute to the observed changes. In addition, the increased need for vitamins both for mother and fetus may account for the observed decline of some vitamins. However, increased catabolism compensated by supplementation does not always prevent these pregnancyinduced changes (Metcoff, 1978). Increased umbilical cord concentrations (riboflavin, vitamin $\mathrm{B}_{6}$ and vitamin $\mathrm{B}_{12}$ ) compared with maternal concentrations suggest active transport to the fetus. Additional longitudinal studies should include hormonal analysis, urinary excretion and umbilical cord concentrations to address the mechanisms of vitamin changes during pregnancy.

Until now, preconceptional and first trimester values were lacking for the vitamins evaluated. Results from the present study suggest that preconceptional concentrations 
of vitamins and their recovery after delivery should be included in the evaluation of pregnancy-induced changes.

\section{Acknowledgement}

The present study was supported by a grant from the Dutch Prevention Fund (NR 28-1358 and NR 28-28760).

\section{References}

Andersson A, Hultberg B, Brattström L \& Isaksson A (1992) Decreased serum homocysteine in pregnancy. European Journal of Clinical Chemistry and Clinical Biochemistry 30, 377-379.

Baker H, Frank O, Thomson AD, Langer A, Munves ED, De Angelis B \& Kaminetzky HA (1975) Vitamin profile of 174 mothers and newborns at parturition. American Journal of Clinical Nutrition 28, 59-65.

Barker DJP, Gluckman PD, Godfrey KM, Harding J, Owens JA \& Robinson JS (1993) Fetal nutrition and cardiovascular disease in adult life. Lancet 341, 938-941.

Boers GHJ, Smals AGH, Trijbels FJM, Fowler B, Bakkeren JAJM, Schoonderwaldt HC, Kleijer WJ \& Kloppenborg PWC (1985) Heterozygosity for homocysteinuria in premature peripheral and cerebral occlusive arterial disease. New England Journal of Medicine 313, 709-715.

Bronstrup A, Hages M, Prinz-Langenohl R \& Pietrzik K (1998) Effects of folic acid and vitamin B-12 on plasma homocysteine concentrations in healthy, young women. American Journal of Clinical Nutrition 68, 1104-1110.

Brown JE (1993) Preconceptional nutrition and reproductive outcome. Annals of the New York Academy of Sciences 678 286-292.

Bruinse HW \& van den Berg H (1995) Changes of some vitamin levels during and after normal pregnancy. European Journal of Obstetrics Gynecology and Reproductive Biology 61, 31-37.

Decker K, Dotis B, Glatzle D \& Hinselman M (1977) Riboflavin status and anemia in pregnant women. Nutrition and Metabolism 21, Suppl. 1, 17-19.

Dunn RT \& Foster LB (1973) Radioassay of serum folate. Clinical Chemistry 22, 1101-1105.

Dyckner T, Ek B, Nyhlin H \& Wester PO (1985) Aggravation of thiamin deficiency by magnesium depletion. A case report. Acta Medica Scandinavica 218, 129-131.

Ek J \& Magnus EM (1981) Plasma and red cell folate during normal pregnancies. Acta Obstetrica et Gynecologica Scandinavica 60, 247-251.

Gal I \& Parkinson CE (1974) Effects of nutrition and other factors on pregnant women's serum vitamin A levels. American Journal of Clinical Nutrition 27, 688-695.

Heller S, Salkeld RM \& Körner WF (1974a) Vitamin B1 status in pregnancy. American Journal of Clinical Nutrition 27, 12211224.

Heller S, Salkeld RM \& Körner WF (1974b) Riboflavin status in pregnancy. American Journal of Clinical Nutrition 27, 12251230.

Hibbard BM (1964) The role of folic acid in pregnancy with particular reference to anaemia, abruption and abortion. Journal of Obstetrics and Gynaecology of the British Commonwealth 71, 529-542.

Kirke PN, Molloy AM, Daly LE, Burke H, Weir DG \& Scott JM (1993) Maternal plasma folate and vitamin B12 are independent risk factors for neural tube defect. Quarterly Journal of Medicine 86, 703-708.

Kloosterman GJ (1970) On intrauterine growth. The significance of prenatal care. International Journal of Gynaecology and Obstetrics 8, 895-912.

Lammer EJ, Chen DT, Hoar RM, Agnish ND, Benke PJ, Braun JT, Curry CJ, Fernhoff PM, Grix AW Jr \& Lott IT (1985) Retinoic acid embryopathy. New England Journal of Medicine 313, 837-841.

Lumeng L, Cleary RE, Wagner R \& Yo PL (1976) Adequacy of vitamin B6 supplementation during pregnancy. A prospective study. American Journal of Clinical Nutrition 29, 1376-1383.

McPartlin J, Halligan A, Scott JM, Darling M \& Weir DG (1993) Accelerated folate breakdown in pregnancy. Lancet 341, 148149.

Metcoff J (1978) Association of fetal growth with maternal nutrition. In Human Growth, vol. 1, pp. 415-460 [F Falkner and JR Tanner, editors]. New York: Plenum Publications Corp.

Mooij PNM, Steegers-Theunissen RPM, Thomas CMG, Doesburg WH \& Eskes TKAB (1993) Periconceptional vitamin profiles are not suitable for identifying women at risk for neural tube defects. Journal of Nutrition 123, 197-203.

Nicols BL \& Nicols VN (1983) Nutrition in pregnancy and lactation. Nutrition Abstracts and Reviews in Clinical Nutrition, Series A 259-273.

Phillips DIW, Barker DJP, Hales CN, Hirst S \& Osmond C (1994) Thinness at birth and insulin resistance in adult life. Diabetologia 37, 150-154.

Rolschau I, Date I \& Kristoffersen K (1979) Folic acid supplementation and intrauterine growth. Acta Obstetrica et Gynecologica Scandinavica 58, 343-346.

Rothman KJ, Moore LL, Singer MR, Nguyen US, Mannino S \& Milunsky A (1995) Teratogenicity of high vitamin A intake. New England Journal of Medicine 333, 1369-1373.

Steegers-Theunissen RPM, Boers GHJ, Blom HJ, Trijbels JMF \& Eskes TKAB (1992) Hyperhomocysteinaemia and recurrent spontaneous abortion or abruptio placentae. Lancet 339, 11221123.

Steegers-Theunissen RPM, Boers GHJ, Trijbels JMF \& Eskes TKAB (1991) Neural-tube defects and derangement of homocysteine metabolism (letter). New England Journal of Medicine 324, 199-200.

Steegers-Theunissen RPM, Renier WO, Borm GF, Thomas CMG, Merkus HMWM, Op de Coul DAW, De Jong PA, van Geijn HP, Wouters M \& Eskes TKAB (1994) Factors influencing the risk of abnormal pregnancy outcome in epileptic women: a multicentre prospective study. Epilepsy Research 18, 261-269.

Steegers-Theunissen RPM, Smithells RW \& Eskes TKAB (1993) Update of new risk factors and prevention of neural tube defects. Obstetrical and Gynecological Survey 48, 287-293.

Steegers-Theunissen RPM, Wathen NC, Eskes TKAB, van RaaijSelten B \& Chard T (1997) Is methionine metabolism important during early human pregnancy? British Journal of Obstetrics and Gynaecology 104, 20-24.

Steen MT, Bodie AM, Fisher AJ, MacMahon W, Saxe D, Sullivan KM, Dembure PP \& Elsas LJ (1998) Neural-tube defects are associated with low concentrations of cobalamin (vitamin B12) in amniotic fluid. Prenatal Diagnosis 18, 545555.

van der Put NMJ, Steegers-Theunissen RPM, Frosst P, Trijbels FJM, Eskes TKAB, Van den Heuvel LP, Mariman ECM, Den Heijer M, Rozen R \& Blom HJ (1995) Mutated methylenetetrahydrofolate reductase as a risk factor for spina bifida. Lancet 346, 505-510.

Von Mandach U, Huch R \& Huch A (1994) Maternal and cord serum vitamin $\mathrm{E}$ levels in normal and abnormal pregnancy. International Journal of Vitamin and Nutrition Research 64, 26-32.

Wang YP, Walsh SW, Guo JD \& Zhang JY (1991) Maternal levels of prostacyclin, thromboxane, vitamin E, and lipid 
peroxides throughout normal pregnancy. American Journal of Obstetrics and Gynecology 165, 1690-1694.

Ziari SA, Mireles VL, Cantu CG, Cervantes M III, Idrisa A, Bobsom D, Tsin AT \& Glew RH (1996) Serum vitamin A, vitamin $\mathrm{E}$, and beta-carotene levels in preeclamptic women in northern Nigeria. American Journal of Perinatology 13, 287291. 